\title{
ON A METHOD FOR SUPPORTING VISUAL IDENTIFICATION OF UNDERWATER OBJECTS
}

\author{
Bogdan Żak, Jerzy Garus
}

Faculty of Mechanical and Electrical Faculty, Polish Naval Academy in Gdynia, Poland

\section{ABSTRACT}

The search and detection of objects under water is carried out by groups of specialised divers. However, their time underwater and their ability to penetrate the depths are limited. For these reasons, the use of unmanned underwater vehicles equipped with technical observation equipment, including TV cameras, is becoming increasingly popular for these tasks. Video images from cameras installed on vehicles are used to identify and classify underwater objects. The process of recognition and identification of objects is tedious and difficult and requires the analysis of numerous sequences of images, and so it is desirable to automate this process. In response to these needs, this article presents the concept of identification of underwater objects based on visual images from an underwater body of water sent from an unmanned underwater vehicle to a base vessel. The methods of initial processing of the observed images from an underwater area as well as the method of searching for selected objects in these images and their identification with the use of the Hough transform will be described. Furthermore, the paper presents the results of the preliminary processing and identification of the observed images following a deconvolution operation.

Keywords: reconnaissance of an underwater area, image processing, identification of objects in video images.

ARTICLE INFO

PolHypRes 2019 Vol. 66 Issue 1 pp. 25 - 34

ISSN: 1734-7009 elSSN: 2084-0535

DOI: $10.2478 /$ phr-2019-0002

Pages: 10, figures: 6 , tables: 0

page www of the periodical: www.phr.net.pl

Publisher

Polish Hyperbaric Medicine and Technology Society

\section{Original article}

Submission date: 03.11.2018 r.

Acceptance for print: 15.12.2018 r. 


\section{INTRODUCTION}

Since the 1970s, there has been a growing interest in underwater vehicles which are being challenged with increasingly complex tasks. Initially, underwater vehicles were used to measure various physical and chemical parameters of sea depths. Currently, one of the main tasks assigned to deep-sea vehicles is to support the search for various types of objects in the water column or lying on the bottom. To this end, vehicles are equipped with a navigation system and sonars allowing for the searching and observing of large underwater areas, as well as vision systems supporting the identification of objects with the use of vision methods [1,2].
The limited size and buoyancy of an underwater vehicle does not always allow for the installation of video analysis devices directly on the vehicle. For these reasons, the sequence of images of an underwater area should be sent through the umbilical cord or acoustic channel to the base ship and only then should the identification process of the researched object be carried out. This process must take place in real time. The image is analysed in the image identification system. During the analysis of the sent images, the search for selected image elements similar to the patterns stored in the identification system database takes place [3].

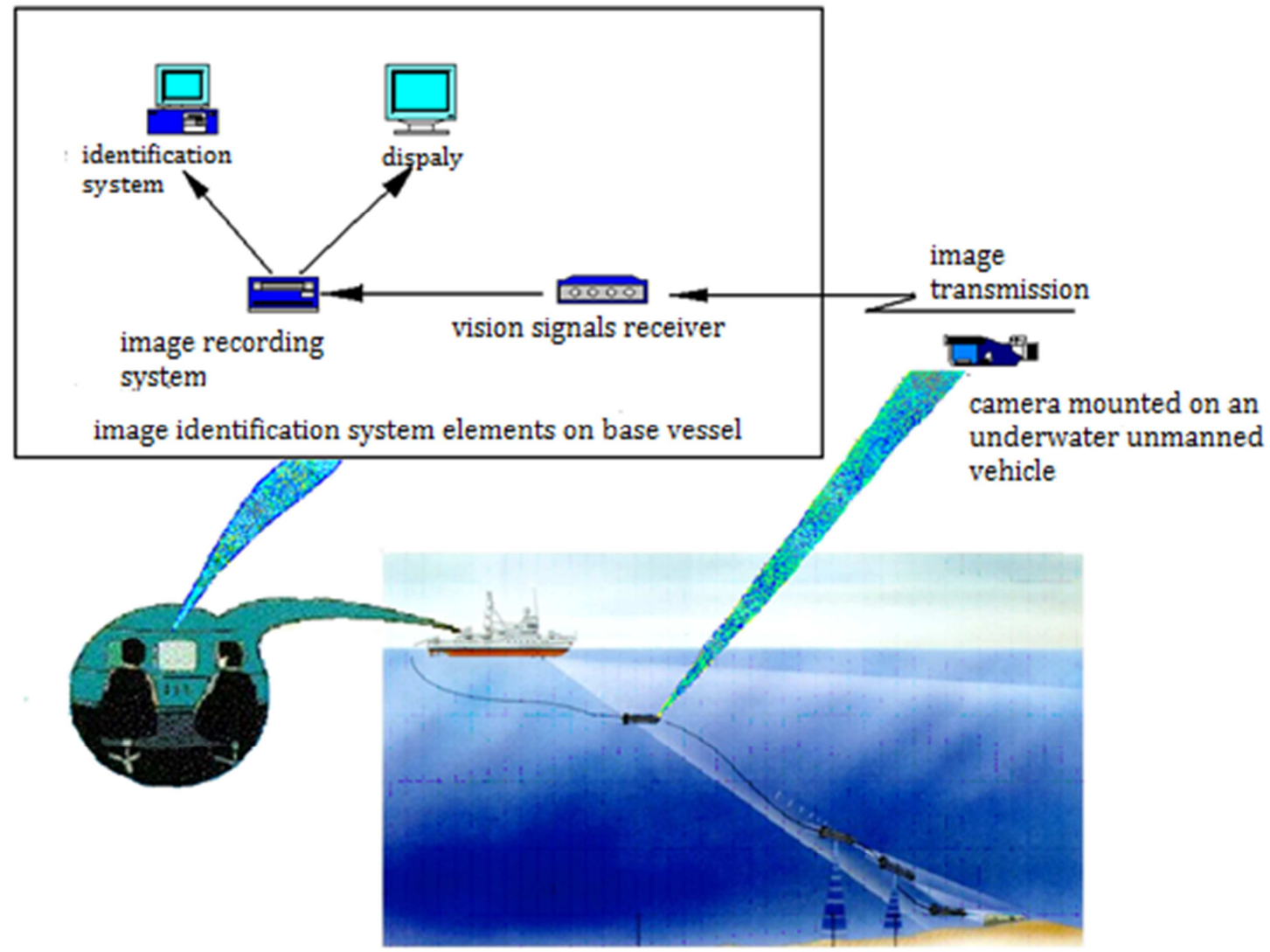

Fig. 1 The concept of a video search and identification system with the use of an underwater vehicle.

The concept of such a solution is presented in Fig. 1. The underwater vehicle is equipped with a camera from which the image is transmitted via a cable track to the operator's console located on the base ship. It is possible to record the video image on the base vessel. The image is analysed in the image identification system. During the analysis, selected image elements similar to the patterns stored in the identification system database are searched for.

\section{INITIAL IMAGE PROCESSING}

Once the image is obtained by the camera, there is a need to improve it or perform other operations on it to ensure the identification of selected elements. In order to improve image quality, a whole range of operations and image transformation methods are used as early as during the initial image processing [4]. The activity of initial image processing is carried out in order to prepare data for final processing, ensure proper image analysis, and, as a result, allow for the identification of selected image elements. In order to improve image quality and enhance details relevant for the identification of an object of interest, filtration is applied during the initial image processing $[5,6]$.

Because of the nature of the aquatic environment and poor lighting in the depths, the image of an underwater object is usually of a very poor quality. It can be improved by compensating for underwater visual disturbances and reducing their impact by reconstructing the original shape of an object. For this purpose, the 
deconvolution method is used to restore the original or intended appearance of the image. The observed image $f(x, y)$ that has deteriorated can be treated as a combination of the initial image $g(x, y)$ with the impulse response function $h(x, y)$ determining what a given element looks like in ideal conditions. Thus, it is possible to write that:

$$
f(x, y)=g(x, y) * h(x, y)
$$

When the Fourier transform is applied, the above equation will take on a form:

$$
F(u, v)=G(u, v) \cdot H(u, v)
$$

Hence, if the impulse response function is known, the image can be reproduced using the equation:

$$
G(u, v)=F(u, v) / H(u, v)
$$

In practice it is required to add additive noise $N(u, v)$ that is present in the image. Thus the above equation will take the following form:

$$
G(u, v)=(F(u, v)-N(u, v)) / H(u, v)
$$
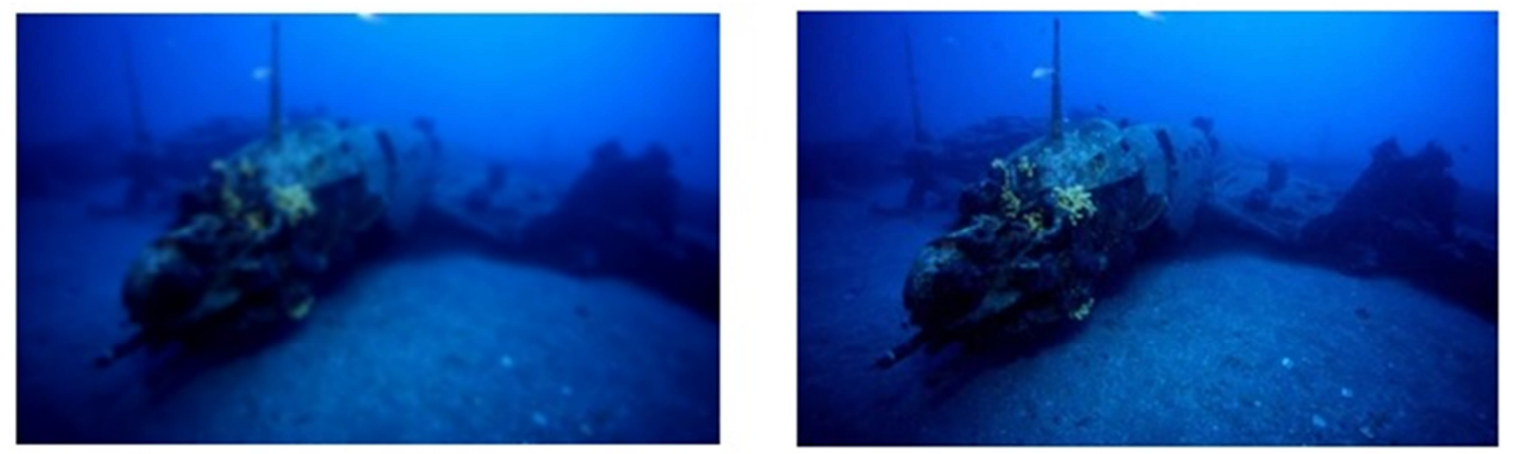

Fig. 2 The effect of deconvolution: a) image of an object lying on the seabed recorded by the TV camera, b) the result of deconvolution on the image of an underwater object recorded by TV camera.

It should be noted that if $H(u, v)$ is small, both expressions in equation (4) may become large and the noise may be amplified. One of the ways to avoid this phenomenon is to use the Wiener filtration, which consists in recording the image in its restored form as:

$$
G(u, v)=[F(u, v) \cdot \hat{H}(u, v)] /\left[N(u, v) \cdot \hat{H}(u, v)+n_{u}\right]
$$

where:

$$
\hat{H}(u, v) \text { - is a complex number coupled for } H(u, v) \text {; }
$$

$n_{u}$ - is the signal-to-noise ratio and the heuristic quantity obtained experimentally.

Fig. 2 presents the result of the deconvolution operation on the image of an underwater object obtained from a TV camera.

\section{IDENTIFICATION OF SELECTED OBJECTS}

\section{ON IMAGES}

The identification of selected elements of video images can be performed using the Hough transform $[7,8]$. The set of colinear points $(x, y)$ can be defined as follows:

$$
\lambda_{0}=\left\{(x, y) \in R^{2}: y-\hat{a} x-\hat{b}=0\right\}
$$

where: $\hat{a}$ and $\hat{b}$ are parameters with values defined by a characterising straight line. The solution to the equation $y-\hat{a} x-\hat{b}=0$ for defined $(\hat{a}, \hat{b})$ is a set of points on the plane.
A special feature of the resulting representation $(\hat{a}, \hat{b}) \rightarrow \lambda_{0}$ is that for one point from the parametric space we obtain a set of collinear points on the image.

In order to determine the values of parameters $(a, b)$ for the straight lines passing through a given point $(\hat{x}, \hat{y})$ it is required to solve the equation $\hat{y}-a \hat{x}-b=0$ in relation to $(a, b)$. This corresponds to the determination of the parameters of a series of lines passing through a given point $(\hat{x}, \hat{y})$. The result of the identification are those straight lines $(a, b)$ which obtain the largest number of votes from all active pixels of the image.

The development of this method for the detection of parametrically defined curves is quite obvious $[9,10]$. The image point $(x, y)$ lying on a curve defined by $n$ parameters $\left(a_{1}, \ldots, a_{n}\right)$ can be represented as a relation:

$$
\lambda_{0}=\left\{(x, y) \in R^{2}: g\left(\left(\hat{a}_{1}, \ldots, \hat{a}_{n}\right),(x, y)\right)=0\right\}
$$

where $g\left(\left(\hat{a}_{1}, \ldots, \hat{a}_{n}\right),(x, y)\right)=0$ is an equation describing a given curve.

To calculate which curve $\left(a_{1}, \ldots, a_{n}\right)$ collects the largest number of votes from active pixels from image $B$, the Hough transform $H\left(a_{1}, \ldots, a_{n}\right)$ is defined as follows:

$$
H\left(a_{1}, \ldots, a_{n}\right)=\sum_{\left(x_{i}, y_{i}\right) \in B} h\left(\hat{x}_{i}, \hat{y}_{i}, a_{1}, \ldots, a_{n}\right)
$$


where:

$h\left(\hat{x}_{i}, \hat{y}_{i}, a_{1}, \ldots, a_{n}\right)=\left\{\begin{array}{l}1 \text { when } g\left(\left(\hat{x_{0}}, \hat{y^{\prime}}\right)_{\mathrm{i}}\left(. a ., a_{n}\right)\right)=0 \\ 0 \text { in the opposite case }\end{array}\right.$

As a result of this procedure an accumulator array is obtained, where each element is identified by the values of parameters $\left(a_{1}, \ldots, a_{n}\right)$. The cell content is increased by 1 when the analytical curve defined by the coordinates of cell $\left(a_{1}, \ldots, a_{n}\right)$ passes through point $(\hat{x}, \hat{y})$ on the object in image $B$. It can therefore be assumed that the Hough transform consists in the mapping of image $B$ on the accumulator array $A$ [11].

The above method can be generalised and extended to irregular patterns. We assume that pattern $W$ may be subject to the rotation transform by angle $\alpha$ and shift by vector $\left[x_{T}, y_{T}\right]$. As a result a given point $\left(x_{i}, y_{i}\right)$ of the pattern is transformed into $\left(x_{i}^{\prime}, y_{i}{ }^{\prime}\right)$. Thus, in the presented case the Hough transform $H\left(x_{T}, y_{T}, \alpha\right)$ for image $B(x, y)$ can be defined as:

$$
H\left(x_{T}, y_{T}, \alpha\right)=\sum_{\left(x_{i}, y_{i}\right) \in M_{w}} h\left(x_{i}, y_{i}, x_{T}, y_{T}, \alpha\right)
$$

when:

$h\left(x_{i}, y_{i}, x_{T}, y_{T}, \alpha\right)=\left\{\begin{array}{l}1 \text { when }\left(x^{\prime}, y^{\prime}\right) \in b(B) \\ 0 \text { in the opposite case }\end{array}\right.$

By generalisation of this method we should adopt the following:

$h\left(x_{i}, y_{i}, x_{T}, y_{T}, \alpha\right)=255-\left|B\left(x_{i}{ }^{\prime}, y_{i}{ }^{\prime}\right)-W\left(x_{i}, y_{i}\right)\right|$
It is a common occurrence that due to the loss of certain information about the image during conversion, the transform can be extended to include the possibility to apply TrueColor palette to images by adding colour distance calculations. The problem consists in calculating the distance between two points $\left(r_{B}, b_{B}, g_{B}\right)$ and $\left(r_{W}, b_{W}\right.$, $\left.g_{W}\right)$ placed in the RGB cube. The distance can be defined as follows:

$$
\left|B\left(x_{i}{ }^{\prime}, y_{i}{ }^{\prime}\right)-W\left(x_{i}, y_{i}\right)\right|=\sqrt{\left(r_{B}-r_{W}\right)^{2}+\left(g_{B}-g_{W}\right)^{2}+\left(b_{B}-b_{W}\right)^{2}}
$$

The presented method in its software implementation can only be used to identify static images. In order to use the above method to identify underwater objects in real time, it should be implemented with the use of additional hardware. This should reduce the processing time of the identification algorithm [12].

\section{SOFTWARE SOLUTION FOR THE IDENTIFICATION OF SELECTED OBJECTS}

The above method was applied to identify underwater objects by a mobile robot using a vision channel. To say that the identification process was a success, the software must answer the following questions: which of the known objects is contained in the image, where it is located and what is its angle of rotation. The software database stores patterns of elements to be identified by the robot. The software obtains the image from the camera placed on the robot through the transmission system shown in Fig. 1. The algorithm of the software operation is shown in Fig. 3.

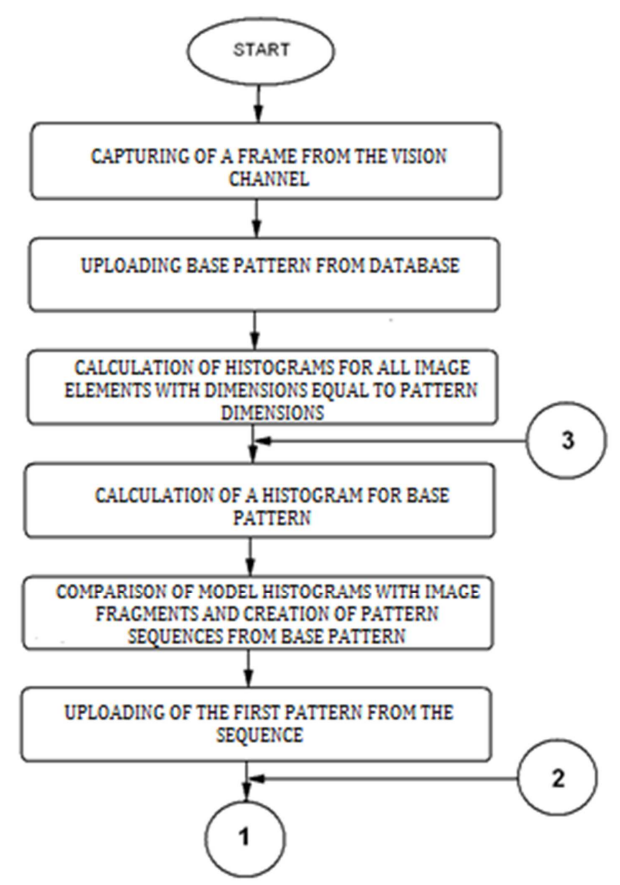

Fig. 3 Underwater object identification algorithm with use of the vision channel of an unmanned underwater vehicle. 


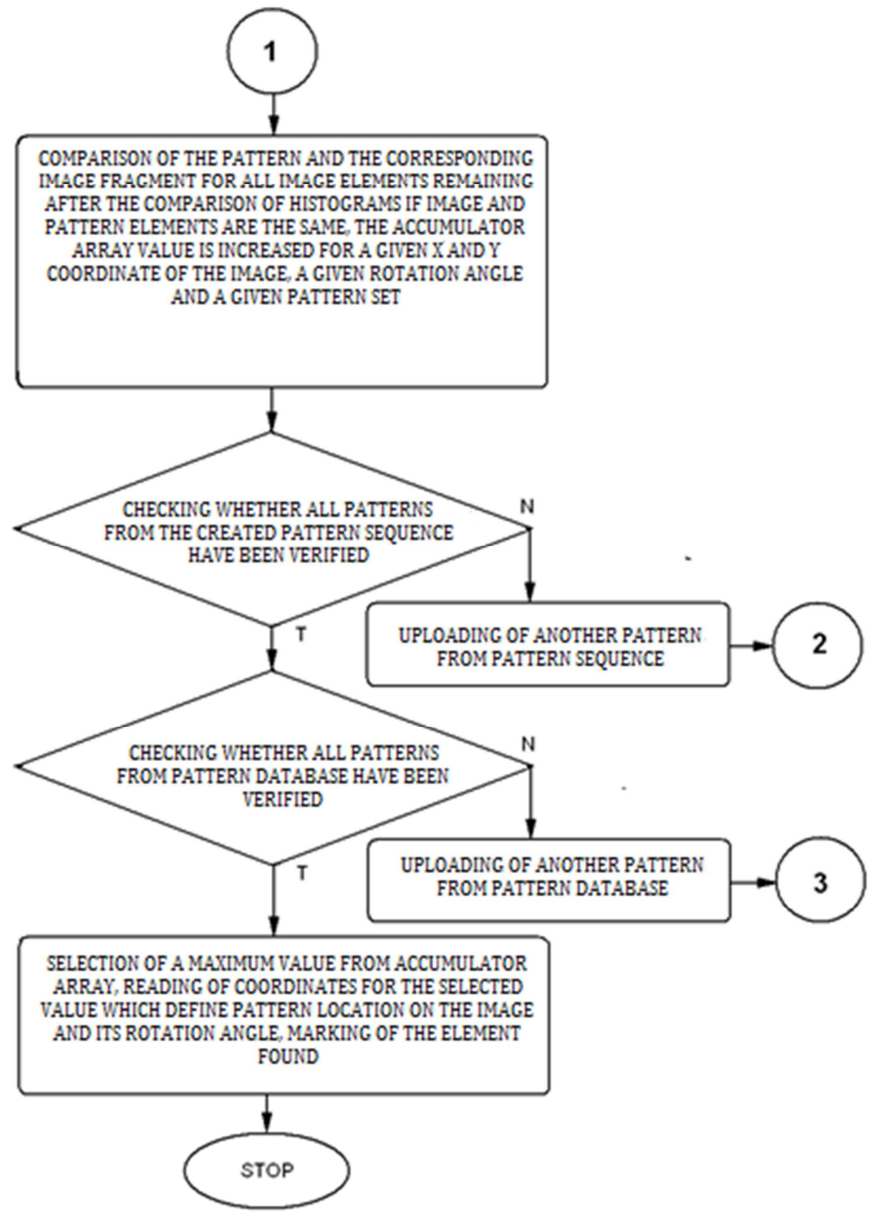

Fig. 3 cont. Underwater object identification algorithm with use of the vision channel of an unmanned underwater vehicle.

In order to reduce the time required to calculate the Hough transform, the set of image elements to be further examined is reduced by means of histogram reduction. The threshold that must be reached during the comparison of histograms was determined experimentally in a manner allowing the lowest possible number of image elements to remain after the reduction, and also in such a way that the element sought would not be omitted. When comparing the pattern and the area, only those elements of the pattern and the selected area of the image that lie inside the circle inscribed in the pattern are compared. The remaining elements are not considered as a result of the performed pattern rotation. In the course of comparison, the pattern elements that match the selected area of the image are counted. Once summed up, they are stored in the accumulator array. This value is identified by the following parameters: pattern application coordinates, pattern rotation angle and pattern number. On their basis we are able to unambiguously determine the type of element and its location in the image, which completes the identification process.

\section{RESEARCH ON THE DEVELOPED} ALGORITHM

The images of an underwater area obtained from the vision channel of an unmanned underwater vehicle were surveyed in accordance with the above method. The examination was carried out on the image from Fig. 2a with no initial processing as well as after applying various filters to the image. The images were used to search for the pattern shown in Fig. 4, which was created from a fragment of the analysed image and entered into the database. The results of the study are shown in Figures 5 and 6.

b)

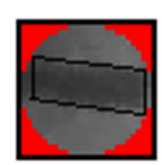

Fig. 4 Pattern sought in the analysed image: a) fragment of the image on the basis of which the pattern was created; b) the created pattern of the object being searched for in the image. 
12

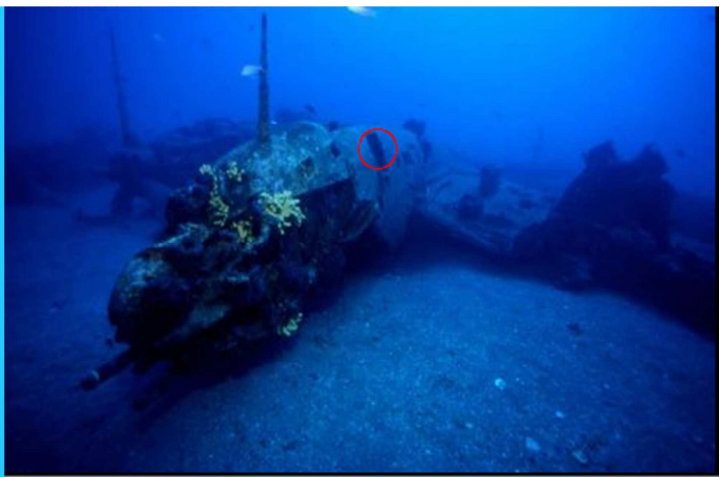

Fig. 5 Pattern identification on the image from fig. 2.a: a) result of application of the Hough transform on the analysed image, b) object identification result.

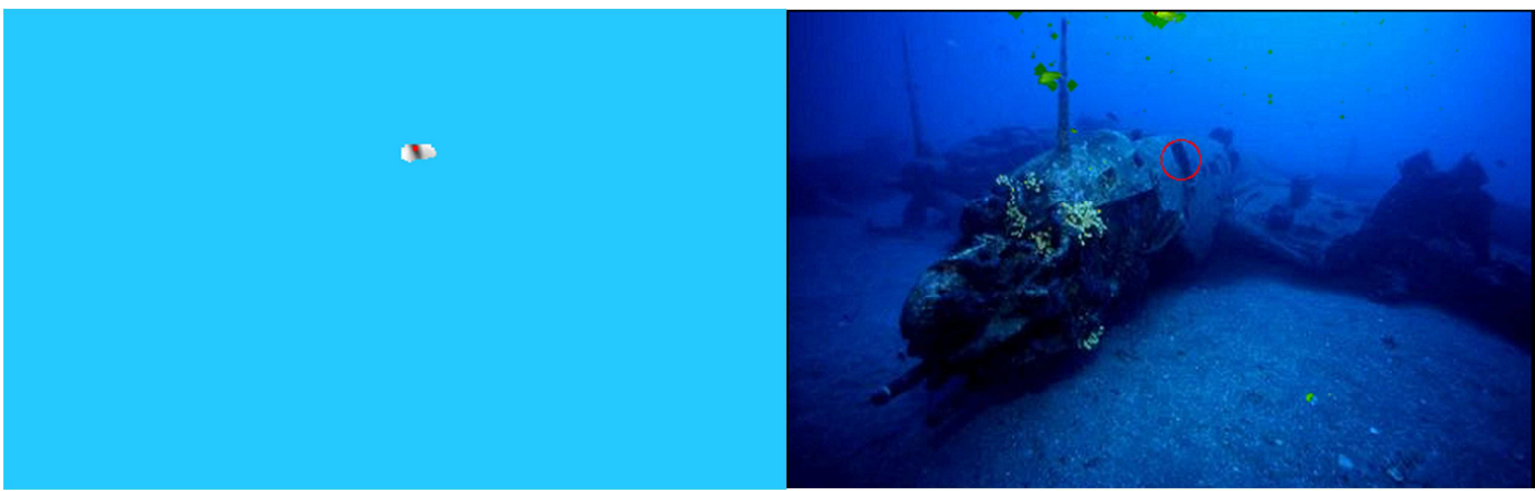

Fig. 6 Pattern identification on the image from fig. 2.b following high-pass filtration a) result of application of the Hough transform, b) object identification result.

\section{Conclusions}

Observation of an underwater area by means of technical image recording is not easy. Interferences occurring in this environment, which include lighting, organic and inorganic contaminants, and the camera's visual acuity, significantly affect the quality of recorded images. Therefore, there is a strong need to process the initial image to allow further operations such as searching for specific items. The presented research results show that the application of simple methods of initial processing allows for a significant improvement of image quality and thus for the enhancement of information significant from the point of view of image recognition and identification.

\section{REFERENCES}

1. Garus J., Kitowski Z.: Sliding control of motion of underwater vehicle. Proc. of the $4^{\text {th }}$ Int. Conference on Marine Technology III ODRA'99, Computational Mechanics Publications, Southampton, 1999, pp. 603-611;

2. Żak B., Hozyń S.: Stereoscopic Technique for a Motion Parameter Determination of Remotely Operated Vehicles, 2016, pp. 263-283, International Conference Mechatronics: Ideas for Industrial Applications, 11-13 May 2015, Gdańsk;

3. Żak B.: Identification of Sunken Objects in Sea Environment Using the Visional System. Polish Journal of Environmental Studies, Vol.18, No 4B, 2009, pp. 231-236;

4. Ballard D. H., Brown C. M.: Computer Vision. Prentice-Hall, Englewood Cliffs, New York 1982

5. Tadeusiewicz R., Korochoda P.: Computer Analysis and Image Processing. Publication Foundation of Progress of Telecommunication, Cracow 1997;

6. Kwiatkowski W: Method of Autoprobes of Standards. IAiR WCY WAT Warsaw 2001;

7. Hough P. V. C.: Method and Means for Recognizing Complex Patterns. U.S. Patent 3,069,654, Dec. 18, 1962;

8. Leavers V. F.: Shape Detection in Computer Vision Using the Hough Transform, Springer, London 1992;

9. Żorski W., Foxon B., Blackledge J., Turner M.: Irregular Colour Pattern Recognition Using the Hough Transform. Bulletin on Institute of Automation an Robotics MUT, 15, 2001, pp. 27-42;

10. Żorski W., Foxon B., Blackledge J., Turner M.: Fingerprint and Iris Identification Method Based on the Hough Transform. Bulletin on Institute of Automation and Robotics MUT, 15, 2001, pp. 43-54;

11. Żorski W., Żak A., Tuner M., Hardware Implementation of the Hough Technique for Irregular Colour and Grey-level Pattern Recognition Biuletyn IAiR WAT 17/2002, Warszawa 2002, pp. 25-44;

12. Żak A., The Concept of Implemention of the Hough Technique in PLD Structures, Materials from the 16th Symposium of the Cybernetics Interest Circle, Warsaw 2000, pp. 125-129. 
dr hab. inż. Bogdan Żak

Akademia Marynarki Wojennej

Wydział Mechaniczno-Elektryczny

81-127 Gdynia

ul. Śmidowicza 69

e-mail: b.zak@amw.gdynia.p

ORCID identifier No: 0000-0003-1010-3911 